\title{
EVALUANDO LOS NUEVOS SISTEMAS DE ACCESO A LA UNIVERSIDAD PARA MAYORES DE 40 Y 45 AÑOS. LA OPINIÓN DE LOS CANDIDATOS
}

\author{
$M^{a}$ del Pilar García Rodríguez \\ Luis Meseguer Martínez \\ Sebastián González Losada \\ Universidad de Huelva
}

\begin{abstract}
RESUMEN: En la actualidad, la aplicación del Espacio Europeo de Educación Superior está significando profundas reformas en el seno del ámbito universitario, teniendo como uno de sus máximos referentes la enseñanza centrada en competencias. Este hecho nos permite el diseño de nuevos sistemas de acceso, uno de ellos mediante la acreditación de experiencia laboral (mayores de 40 años), y un segundo para aquellos que en ausencia de dicha experiencia, deseen continuar estudiando (mayores de 45 años).

Este artículo tiene como objetivo describir y evaluar los primeros resultados de la aplicación de los sistemas que se han diseñado para el acceso de los mayores de 40 y 45 años en Huelva, desde dos perspectivas. Una general basada en los datos de presentados, aprobados, titulaciones y admitidos; y una segunda en la que se analizan las expectativas y motivaciones de los candidatos presentados en Huelva, a través de una encuesta. Los resultados de esta primera convocatoria han sido muy satisfactorios y sorprendentes en cuanto a número de candidatos. Las valoraciones han sido positivas por parte de los mismos, hallando diferencias significativas en función de los procedimientos, sexo y expectativas.
\end{abstract}

PALABRAS CLAVE: Requerimientos para acceder a la Universidad, estudiantes mayores de 40 y 45 años, educación continua, aprendizaje a lo largo de la vida.

\section{ASSESING NEW SYSTEMS OF ENTRANCE TO UNIVERSITY FOR ADULTS PEOPLE OVER 40 AND 45. THE CANDIDATES' OPINIONS}

ABSTRACT: The incorporation of our educative system to the European Space of Higher Education has implied a deep change in our university 
curricula. Education in competences has become the key to develop our programs. It also enables us to create new ways to university entrance through the certification of work experience and validation of competences (for adults over 40), or without experience (for adults over 45).

Our report aims at describing and analyzing the results of accessing university process which have been designed and put into practice in Huelva for people over 40 and 45 . In addition to this, we would like to describe their views of the systems which have been carried out. The results have been very satisfactory and surprising not only by the number of candidates but also by the positive candidates assessments.

KEYWORDS: Degree requirements in Higher Education, adult students over 40 and 45, continuing education, life-long learning.

Recibido: $11 / 08 / 2011$

Aceptado: 19/10/2012

\section{INTRODUCCIÓN}

Las universidades españolas como consecuencia de la Ley 4/2007 y el Real Decreto 1892/2008 (Ministerio de Educación, 2007 y 2008 respectivamente), regulan por primera vez en su historia dos nuevas vías de acceso para el colectivo de adultos que no reúnan condiciones de acceso a la universidad. Estas vías eran necesarias puesto que la única posibilidad existente hasta el curso 2009-2010 para aqueIlas personas mayores que no tuvieran ningún título habilitante para acceder a la institución de enseñanza superior, eran las pruebas de Acceso a la Universidad para Mayores de 25 años.

La nueva legislación ha establecido dos vías. Una para personas mayores de 40 años que puedan acreditar experiencia laboral y profesional, y una segunda fórmula para los que en ausencia de dicha experiencia, tengan 45 años o más. En el caso del sistema de mayores de 40 se lleva a cabo una evaluación de la experiencia profesional y laboral y una entrevista, y el acceso se establece para determinadas titulaciones que guarden relación con la experiencia laboral acreditada. Para el sistema de acceso de mayores de 45, se realiza una prueba adaptada con dos ejercicios (un comentario de texto o tema de actualidad y otro de Lengua Castellana) y una entrevista para apreciar la madurez e idoneidad de los candidatos. En este caso el acceso tiene carácter general para todas las titulaciones.

Los sistemas diseñados por las universidades españolas han sido heterogéneos sobre todo en el caso del sistema para mayores de 40 años y han requerido de grandes esfuerzos y tiempo para su puesta en marcha ${ }^{1}$. Además, ésta ha sido la primera

1. En la Memoria Final del Proyecto hemos recogido todos los sistemas diseñados en España así como los datos de la acogida de la primera convocatoria. Proyecto Financiado en la Convocatoria de 2009 del Programa de Estudios y Análisis del Ministerio de Educación. Resolución de 20 de agosto de 2009; código EA2009-0156. "Análisis y propuesta de sistemas de certificación de competencias y adaptación de pruebas de acceso para mayores de 40 y 45 años". Nuestro agra- 
vez que las universidades han contemplado el reconocimiento del aprendizaje adquirido a través de la experiencia profesional para el acceso a sus titulaciones. Esta nueva vía se aleja de las fórmulas tradicionales de acceso, sobre las que se han realizado multitud de estudios e investigaciones tales como los de Muñoz Vitoria, 1993; 1995; diversos autores en el monográfico de Revista de Educación de 1997, etc.

De las 34 Universidades públicas que implantaron este sistema para mayores de 40 en la primera convocatoria, es destacable el caso de las nueve universidades andaluzas ya que consiguieron acordar y regular un procedimiento de acceso común. En el caso del sistema de acceso para mayores de 45, su diseño y puesta en marcha no ha sido difícil, ya que las Universidades lo han equiparado al sistema de mayores 25 celebrando conjuntamente las pruebas para estas dos vías de acceso salvo las Universidades de Santiago de Compostela y Vigo (que prepararon exámenes distintos para cada vía de acceso) y la de Las Palmas de Gran Canaria que no celebró esta prueba en la primera convocatoria.

El sistema diseñado ${ }^{2}$ en el caso del acceso para mayores de 40 que fue adoptado por todas las Universidades Andaluzas, se basó en la evaluación de la experiencia en relación a la titulación universitaria que solicitaba el candidato y una entrevista. Para la primera fase en la que se valoró la experiencia laboral y profesional, se construyó una tabla de afinidades entre estudios universitarios y Familias Profesionales partiendo de las Memorias de verificación, las competencias expresadas en los Libros Blancos de la ANECA (2008) y el Informe Tuning (González y Wagenaar, 2003). Las experiencias profesionales fueron clasificadas a partir de las Cualificaciones por familias y niveles de competencias establecidas por el Instituto Nacional de las Cualificaciones (INCUAL, 2009)3. Para valorar el tiempo de experiencia, se diseñó una fórmula partiendo de una tabla de equivalencias entre niveles de competencia del INCUAL y los grupos de cotización de la seguridad social y se aplicó la fórmula: Puntuación = Tiempo de experiencia $\times \mathrm{T} \times \mathrm{C}$ siendo " $\mathrm{T}$ ", el valor de la experiencia laboral y profesional (T equivaldrá a 1,25, cuando se trate de un año completo de trabajo y 0,104 en caso de meses completos o periodos de más de 15 días) y " $C$ " el coeficiente según nivel de competencia (cuando el nivel de competencia es 3 , el valor será de 1 ; un nivel 2, el valor será de 0,8 ; y cuando el nivel de competencia es 1 , el valor será de 0,6). El baremo propuesto garantiza un mínimo de años de experiencia para considerar a un candidato apto en la Fase I (siempre que el valor sea, al menos de 5 puntos). Las puntuaciones no tienen limitación alguna y permite ordenar a los candidatos empleando para ello criterios objetivos y transparentes para los posteriores procesos de admisión. En el caso de las entrevistas (fase II), fueron desarrolladas por especialistas en recursos humanos, tuvieron una duración de unos 20 minutos y una orientación general a valorar el

decimiento por dicha financiación, así como al apoyo que hemos recibido por parte de la Comisión de Distrito Único Andaluz que ha hecho posible la puesta en marcha de los sistemas que diseñó este equipo de investigación.

2. Por los miembros de este equipo de investigación.

3. Dicha tabla fue presentada en la RUNAE de noviembre de 2009 y publicada a través de Distrito Único Andaluz para su difusión (http://www.juntadeandalucia.es/innovacioncienciayempresa/sguit/documentacion/TITULACIONES_FAMILIAS.pdf). 
grado de madurez e idoneidad respecto a los estudios universitarios a los que el/la aspirante deseaba acceder, por lo que los referentes fueron las competencias básicas expresadas en las Memorias de Grado.

En este trabajo presentamos los resultados obtenidos por los candidatos a las pruebas de mayores de 40 años con experiencia profesional y por los mayores de 45 que se examinaron, participando en la primera convocatoria que se celebraba de ambos sistemas de acceso en el curso 2009-2010 en su aplicación en la Universidad de Huelva ${ }^{4}$. Para evaluar estos primeros resultados se describen los datos de presentados, aprobados, admitidos y titulaciones a las que han accedido; y se recogen datos parciales de una encuesta ${ }^{5}$ que se aplicó a todos los candidatos con la que se trata de conocer (entre otros aspectos), las expectativas y motivaciones de los candidatos presentados en Huelva por los estudios universitarios.

Las motivaciones han sido estudiadas en el ámbito de la psicología y se suelen agrupar en torno a metas relacionadas con la tarea, con el ego, con la valoración social y con la consecución de recompensas externas (Ausubel, 1981). Las expectativas de éxito o autoeficacia han sido estudiadas como un factor condicionante del aprendizaje y el rendimiento con modelos como el de Pintrich y Schrauben (1992) y que han sido replicados con trabajos como los de Martínez y Galán (2000), Roces, Tourón y González (1995) y Cardozo (2008) entre otros. En nuestro estudio nos interesaba conocer cuáles eran sus motivaciones y expectativas de éxito al inicio, como punto de partida para valorar los sistemas, así como prever el posible éxito de estos estudiantes mayores.

\section{MÉTOdo}

\section{Participantes}

Para hacer una valoración del éxito, en términos de participación y acceso-admisión a la Universidad de Huelva, empleando los nuevos sistemas de acceso para mayores, se han recopilado las estadísticas de la convocatoria, analizando la documentación oficial de dicha institución.

Para recabar las valoraciones de los participantes respecto a los sistemas de acceso se aplicó una encuesta a la totalidad de los candidatos. 86 sujetos de los que 58 se presentaron a la evaluación de la experiencia profesional (sistema para mayores de 40 años), y 28 a los exámenes del sistema de mayores de 45.

4. La descripción de los sistemas diseñados en las universidades españolas pueden encontrarlo en García Rodríguez, M.P., Meseguer Martínez, L., González Losada, S. y Pozo Muñoz, C. (2012). ¿Cómo acceden los Mayores a la Universidad en España? Nuevas oportunidades para la formación a lo largo de la vida. Revista Española de Pedagogía no 253, 515-535. Asimismo, los resultados de la aplicación de dichos sistemas por las Universidades Andaluzas en la primera convocatoria, se encuentran publicados (en prensa). La referencia es: García, M.P., Meseguer, L., González, S. y Barrera, A.R. (2014). Aprendizaje a lo largo de la vida: éxito y futuro del sistema de acceso a la Universidad para Mayores de 40 y 45 años en Andalucía. Revista de Educación, 363. DOI: 10.4438/1988-592X-RE-2012-363-172.

5. Encuesta incluida en el anexo a este documento. 


\section{Procedimiento}

La elaboración de la encuesta estuvo guiada por los juicios de varios expertos en evaluación de programas y satisfacción, así como por las respuestas a varias entrevistas en profundidad realizadas a directores y personal técnico de acceso de diversas universidades. Las encuestas aplicadas fueron dos: una para evaluar el sistema de acceso de mayores de 40 años y la otra para el sistema de acceso para mayores de 45. Los bloques de contenido de la primera encuesta fueron:

(a) datos identificativos (sexo, edad, familia, empleo y nivel de estudios).

(b) motivaciones y expectativas para realizar el acceso a la universidad (12 ítems).

(c) preferencias respecto a la elección de los estudios universitarios y la información de que disponen de ellos (9 ítems).

(d) valoración respecto a la difusión de los sistemas, aspectos organizativos y de gestión del procedimiento (15; siendo dos de ellos preguntas abiertas para que expresen su opinión global, así como las sugerencias que estimen oportunas).

El instrumento diseñado para los mayores de 45 años, constaba de estos mismos bloques de contenido, aunque en el cuarto apartado, el procedimiento a evaluar se refería a los exámenes a los que se sometieron (ítem 13), siendo tan solo una la pregunta de carácter abierto. Por tanto, la estructura y contenido fueron muy similares en ambos procesos de encuestación.

En ambos instrumentos, el encuestado debía valorar en una escala tipo Likert de 4 puntos (siendo 1 un valor mínimo y 4 un acuerdo en grado máximo), respecto a cuáles habían sido los motivos que le llevaron a realizar el acceso a la universidad y en general, cuáles son sus opiniones y valoraciones sobre los respectivos procesos de acceso.

Puesto que según el Real Decreto 1892/2008 y nuestra propia propuesta, los candidatos debían realizar una entrevista personal, se consideró idóneo pasar la encuesta tras la celebración de la misma. La aplicación fue coordinada por miembros del equipo de investigación, que se encargaba de explicar la finalidad de la encuesta y garantizar el anonimato. La administración de la misma se realizó en el mes de mayo de 2010, fechas en las que se estaban desarrollando las entrevistas. El porcentaje de participación fue superior al 90\% de la población para ambas vías de acceso.

Hemos realizado análisis descriptivos de carácter cuantitativo empleando el SPSS. En la mayoría de las variables hemos recodificado las respuestas utilizando la agrupación de dicotomías, para obtener datos que sinteticen la realidad estudiada.

\section{Resultados}

\section{Resultados de la convocatoria}

La demanda de estudios universitarios por parte de la población adulta andaluza ha ido incrementándose en los últimos años, especialmente en los últimos dos cursos académicos. Como puede observarse en la Tabla 1, entre 2006 y 2010 casi 
se ha triplicado el número de adultos que pretenden acceder al sistema universitario andaluz. Los incrementos mayores se dan entre 2008 y 2009 (57,8\%) y entre 2009 y 2010 (81,9\%). Hay que tener en cuenta, sin embargo, que en 2010 ya se habían implantado los dos nuevos sistemas de acceso para adultos. Si sólo tenemos en cuenta la prueba de mayores de 25 años, el incremento de inscritos en 2010 sería sólo de 884 adultos $(27,2 \%)$. El resto del incremento registrado ese año se produjo por los inscritos en los nuevos sistemas de acceso: 1342 en mayores de 40 años y de 434 en mayores de 45.

Tabla 1. Número de inscritos en el sistema de acceso para mayores de 25 años (2006-2010) y en los nuevos sistemas de acceso para adultos (2010)

\begin{tabular}{|c|c|c|c|c|c|c|c|}
\hline \multicolumn{8}{|c|}{ Evolución de los candidatos en la Universidad de Huelva } \\
\hline 2006 & 2007 & 2008 & 2009 & \multicolumn{4}{|c|}{2010} \\
\hline M25 & M25 & M25 & M25 & M25 & M40 & M45 & Total \\
\hline 146 & 142 & 164 & 275 & 329 & 58 & 28 & 415 \\
\hline \multicolumn{8}{|c|}{ Evolución de los candidatos en las Universidades Públicas Andaluzas } \\
\hline 2006 & 2007 & 2008 & 2009 & \multicolumn{4}{|c|}{2010} \\
\hline M25 & M25 & M25 & M25 & M25 & M40 & M45 & Total \\
\hline 1992 & 1919 & 2059 & 3.249 & 4.133 & 1.342 & 434 & 5.909 \\
\hline
\end{tabular}

La misma tendencia se observa en los presentados a las pruebas de acceso para adultos en la Universidad de Huelva. Los mayores incrementos en el número de inscritos también se produjeron en los años 2009 (40,4\%) y 2010 (50,9\%), aunque estos incrementos fueron inferiores a los registrados como promedio en el resto de las universidades andaluzas. Igualmente, el incremento del año 2010 se distribuyó de forma desigual, con 54 inscritos más en la prueba de mayores de 25 años y con 86 inscritos en las nuevas pruebas.

En cuanto al éxito de los candidatos, los resultados muestran que la mayoría de los adultos presentados en la Comunidad Autónoma Andaluza consiguieron superar las pruebas establecidas en los nuevos sistemas de acceso $(80,7 \%)$. Hay que señalar, sin embargo, que la tasa de éxito fue claramente diferente en las pruebas de mayores de 40 años (88,6\% de aprobados) que en las pruebas de mayores de 45 años (56,2\% de aprobados). Se encontraron importantes diferencias de género en la tasa de éxito $(83,8 \%$ de hombres frente a un $77 \%$ de mujeres). 
EVALUANDO LOS NUEVOS SISTEMAS DE ACCESO A LA UNIVERSIDAD PARA MAYORES DE 40 Y 45 AÑOS...

Tabla 2. Número de candidatos presentados y porcentaje de aprobados en la convocatoria de 2010 en los Sistemas de Mayores de 40 y 45 años en Huelva y Andalucía por sexo

\begin{tabular}{|l|c|c|c|c|c|c|c|c|c|c|c|c|}
\hline & \multicolumn{4}{|c|}{ Mayores de 40 años con experiencia laboral. Año 2010 } & \multicolumn{5}{c|}{ Mayores de 45 años. Año 2010} \\
\hline & \multicolumn{3}{|c|}{ Presentados/as } & \multicolumn{4}{|c|}{ Aprobados/as } & Presentados/as & \multicolumn{3}{c|}{ Aprobados/as } \\
\hline Universidad & Mujeres & Hombres & Mujeres & $\%$ & Hombres & $\%$ & Mujeres & Hombres & Mujeres & $\%$ & Hombres & $\%$ \\
\hline UHU & 33 & 25 & 24 & $\mathbf{5 8 , 5}$ & 17 & $\mathbf{4 1 , 5}$ & 16 & 11 & 9 & $\mathbf{5 2 , 9}$ & 8 & $\mathbf{4 7 , 1}$ \\
\hline Andalucía & 559 & 783 & 474 & $\mathbf{3 9 , 9}$ & 715 & $\mathbf{6 0 , 1}$ & 255 & 179 & 153 & $\mathbf{6 2 , 7}$ & 91 & $\mathbf{3 7 , 3}$ \\
\hline
\end{tabular}

En la Tabla 2 se reflejan el número de aprobados en las pruebas de los nuevos sistemas de acceso, para el total de las universidades andaluzas y, específicamente, para la Universidad de Huelva. Los resultados en la Universidad de Huelva arrojan una mayoría de aprobados $(68,2 \%)$, aunque claramente inferior a la obtenida en el conjunto de las universidades andaluzas. Diferenciando en función del tipo de pruebas, la tasa de éxito en la prueba de mayores de 40 años fue superior a la obtenida en la prueba de mayores de 45 años también en el caso de la Universidad de Huelva $(70,7 \%$ frente a $59,3 \%)$. Las diferencias en las tasas de éxito son, en cambio, prácticamente inexistentes diferenciando los resultados por sexo. En el caso de la Universidad de Huelva $(69,4 \%$ para los hombres y $67,3 \%$ para las mujeres).

Finalizadas las pruebas, el colectivo de candidatos que las habían superado y que, por tanto, podían solicitar plaza en alguna de las titulaciones de las ofertadas en el curso 2010-2011, estaba formado por 1.433 personas en el conjunto de las universidades andaluzas, de las cuales 58 habían realizado las pruebas en la Universidad de Huelva.

En aplicación de la Resolución de la Comisión de Distrito Único (Junta de Andalucía, 2010), que estableció que un 2 por ciento de las plazas quedarían reservadas para los que superaran los procedimientos de 40 ó 45 años, un total de 1.036 plazas fueron asignadas inicialmente para ambos cupos. En la práctica, y con los redondeos al alza la oferta para este cupo fue de 1.200 plazas, de las que finalmente sólo fueron adjudicadas 603 plazas (50,2\%). En el caso de Huelva, las plazas ofertadas fueron 66, de las cuáles se adjudicaron 32 plazas (48,5\%).

Observando estos datos vemos que, a priori, la oferta a nivel de Comunidad no es suficiente para atender la demanda, existiendo un déficit inicial de 233 plazas. En cambio, en la Universidad de Huelva existía un superávit inicial de 8 plazas. Estos cálculos son puramente teóricos, puesto que parten del supuesto de que la demanda se distribuye homogéneamente entre las distintas titulaciones. Evidentemente este reparto homogéneo no se produce, ya que finalmente han quedado plazas libres (597 en el conjunto de las universidades andaluzas y 34 en la Universidad de Huelva), y candidatos aprobados sin plaza adjudicada (830 candidatos en el conjunto de las universidades andaluzas y 26 en la Universidad de Huelva).

En general, las titulaciones más demandadas han sido las de la Rama de conocimiento de Ciencias de la Salud, Humanidades y Ciencias Sociales. Esta tendencia ha sido generalizada en Andalucía. En el caso de Huelva, las titulaciones que han 
cubierto su oferta para estos cupos han sido: Administración y Dirección de Empresas, Derecho, Educación Social, Enfermería, Filología Hispánica, Historia, Ingeniería Agrícola, Relaciones Laborales y Recursos Humanos.

\section{Resultados de las valoraciones de los candidatos al acceso de 40 y 45 años}

Los candidatos a las pruebas de acceso para mayores de 40 años que superaron la fase de valoración de la experiencia profesional fueron 41, de los cuales 38 accedieron a contestar la encuesta de valoración $(92,7 \%)$. La edad de estos candidatos oscilaba entre los 40 y los 62 años, con una mayoría entre 40 y 50 años $(73,68 \%)$. Había un mayor porcentaje de mujeres (casi el 57\%) que de hombres. La mayoría de los candidatos tenían hijos a su cargo (80\%) y estaban trabajando en el momento de la encuesta $(86,8 \%)$.

Con respecto al nivel de estudios previo de los candidatos, había un mayor porcentaje de sujetos con estudios de Formación Profesional o Bachillerato (63,2\%) que con sólo estudios de Primaria o Graduado escolar. El 71,1\% tenían conocimientos de otro idioma (inglés o francés). Igualmente, aunque la mayoría tenía conocimientos de informática sólo a nivel de usuario (76,3\%), nos encontramos con el 23,7\% con conocimientos a nivel de experto.

Los 17 candidatos que en el sistema de acceso para mayores de 45 años superaron la primera fase accedieron a participar en la encuesta de valoración. Al igual que en el caso de las pruebas para mayores de 40 años, existía un porcentaje ligeramente mayor de mujeres que de hombres y casi el $80 \%$ tenían hijos a su cargo. A diferencia de lo encontrado en los mayores de 40 años, existía un mayor número de candidatos con estudios primarios que con estudios secundarios (11 candidatos frente a 9), y sólo trabajaban en el momento de la encuesta 8 candidatos. El 73,7\% tenía conocimientos de otro idioma (inglés o francés). En cuanto a los conocimientos de informática, el 84,2\% tenían conocimientos a nivel de usuario, un candidato tenía conocimientos a nivel de experto, y dos candidatos no tenían ningún conocimiento.

Las expectativas de éxito en ambos grupos eran muy altas. Para los mayores de 40 años, un 52,6\% esperaban aprobar todas las asignaturas, mientras que un 44,7\% creía que solo aprobaría la mitad y un 2,6\%, consideraba que sólo superaría menos de la mitad de las asignaturas. Para los mayores de 45 años, un 63,2\% esperaban aprobar todas las asignaturas, un $21,1 \%$ sólo la mitad y un 15,8\% menos de la mitad de las materias.

Las motivaciones que tienen los candidatos que pretenden acceder a la Universidad por el sistema de acceso para mayores de 40 años, pueden ser ordenadas en función de su importancia desde aquellas elegidas por todos los candidatos -la mejora de sus opciones profesionales y la profundización en sus conocimientos (100\%)-, seguido por otras motivaciones que son elegidas mayoritariamente -el crecimiento personal $(94,7 \%)$, los deseos de seguir estudiando $(81,6 \%)$, el prestigio de tener una titulación universitaria $(73,7 \%)$, el estar activo mentalmente $(71,1 \%)-$, y, por último, aquellos motivos que sólo son elegidos por una minoría de los candidatos -el establecimiento de nuevas relaciones $(31,6 \%)$ y los consejos de sus familiares $(28,9 \%)$ -

Los resultados en cuanto a los motivos expresados por los candidatos presentados a las pruebas para mayores de 45 años, presentan una mayor variabilidad. 
Según sus respuestas se puede señalar que lo que les mueve a estudiar es fundamentalmente la mejora de sus conocimientos y su crecimiento personal $(84,2 \%$ en ambos casos), seguido por el deseo de estar activos mentalmente $(73,3 \%)$ y el deseo insatisfecho de haber continuado los estudios $(63,2 \%)$. La mejora de las opciones profesionales queda relegada a un quinto puesto, probablemente derivado del hecho de que no trabajan más que el $40 \%$ de los encuestados. El deseo de establecer relaciones $(47,4 \%)$ es un motivo elegido en mayor proporción que en el grupo de mayores de 40 . Y por último, a los mayores de 45 no parece influirles especialmente el prestigio de tener una titulación universitaria $(26,3 \%)$, ni los consejos de sus familiares ni amigos (15,8\%).

La gran mayoría se decanta por una titulación concreta, sin embargo cuando valoran el nivel de información que poseen respecto a dicha opción de estudios, reconocen no tener muchos conocimientos. En la encuesta les preguntamos en qué grado conocían los planes de estudio, niveles de exigencia, características de la carrera y salidas profesionales. Las respuestas a estas preguntas se presentan resumidas en la tabla 3.

Tabla 3. Percepción de los candidatos sobre su conocimiento de las titulaciones que quieren cursar

\begin{tabular}{|l|c|c|}
\hline & Mayores de 40 & Mayores de 45 \\
\hline Conoce el plan de estudios & $42,1 \%$ & $21,0 \%$ \\
\hline Conoce el nivel de exigencia & $47,3 \%$ & $42,1 \%$ \\
\hline Conoce las salidas profesionales & $63,1 \%$ & $42,1 \%$ \\
\hline Considera que es fácil y de duración adecuada & $42,1 \%$ & $26,4 \%$ \\
\hline
\end{tabular}

Destacamos como dato interesante, el que los mayores de 45 perciban el reto de los estudios de manera más optimista que los mayores de 40, al valorar en mayor medida las titulaciones como fáciles y de duración adecuada. Es posible que el carácter intrínseco de su motivación -menos determinado por la opinión de amigos o familiares- pueda condicionar su propia visión de los estudios.

Por otra parte, se preguntó a los candidatos cuál era su opinión acerca la difusión realizada por la Universidad de Huelva de las nuevas pruebas de acceso. En ambos grupos destacan el papel relevante de la web institucional de la Universidad de Huelva, así como del personal que les había informado.

Por último, referirnos a las valoraciones de los sistemas de acceso. En el grupo de mayores de 40 años hay un 63,2\% que dicen estar bastante o muy de acuerdo con el sistema de baremación. Respecto a las entrevistas, las valoran muy positivamente $(78,9 \%)$, como método de evaluación de la madurez de su experiencia. No se encontraron diferencias relevantes en las respuestas de los mayores de 45 años. Mayoritariamente en el caso de los mayores de 40 años (76,3\%), y la mitad de los mayores de 45 años, creen necesario crear un sistema de orientación específico para ellos. 


\section{DisCUSIÓN}

Se pueden identificar multitud de razones para justificar la necesidad y urgencia de estas nuevas vías de acceso a la enseñanza universitaria. El desempleo se ha incrementado considerablemente en los últimos años, siendo una de las tasas más altas de la Unión Europea (desde septiembre de 2011 hasta septiembre del 2012 el paro se sitúa en España en torno al 25,1\% $\%^{6}$. La última encuesta de Población activa realizada por el Instituto Nacional de Estadística del tercer trimestre de 2011, sitúa la tasa de paro nacional en el 21,52\%, y la andaluza en el 30,93\% (Instituto Nacional de Estadística, 2011), siendo mayores en el caso de las mujeres y estando inversamente relacionada con el nivel formativo (Ministerio de Educación, 2010). Como muestra, el 31,92\% de las personas cuyo nivel de formación obtenido son los estudios primarios están en paro, mientras que el 12,82\% de los que poseen estudios universitarios, están en esta situación (Instituto Nacional de Estadística, 2012). Superamos claramente la media europea respecto al abandono escolar y tenemos una tasa de fracaso del 32\% (Ministerio de Educación, 2010), sin embargo, estudios recientes marcan un descenso de casi 5 puntos (26,5\% de abandono en $\left.2011^{7}\right)$.

La demanda de estudios por parte de la población adulta está incrementándose considerablemente. El porcentaje de la población adulta española (25 a 64 años) que había accedido a Educación Secundaria Postobligatoria o Educación Superior se incrementó del 32\% al 51\% en el periodo 1997-2007 (Ministerio de Educación y Consejo Escolar, 2010). Teniendo en cuenta que estos casos son anteriores a la crisis económica, es de suponer que la demanda formativa va a experimentar un aumento en los próximos años. Los motivos de este aumento son explicados desde diversas perspectivas. Rué (2003-2004), considera que puede deberse al aumento del nivel de vida de la población implicando a su vez el incremento de sus expectativas formativas y la necesidad de adaptación de las nuevas exigencias del actual y cambiante mercado de trabajo. Frente a esta postura, Trinidad (2005) considera que el aumento se podría explicar por las previsiones de descenso de la natalidad, de ahí que se pueda esperar que la demanda de estudios universitarios provenga en mayor medida de personas mayores con motivos de ocio o parados que "credencialmente" acumulen títulos con el fin de mejorar las perspectivas de empleo. En esta misma línea de actualización de conocimientos o reciclaje profesional para la mejora de la empleabilidad se posiciona Pérez Díaz (2001).

Por tanto, estas nuevas vías de acceso a la Universidad van a contribuir a la promoción de nuevas estrategias de aprendizaje a lo largo de la vida, que la Comisión Europea recomendaba (2003), así como a la formación continua, posibilitando la mejora de la empleabilidad y produciendo una diversificación de la población estudiantil universitaria. En este sentido, podríamos afirmar que estaríamos en términos de Zabalza (2003-2004), frente a innovaciones referidas a las relaciones entre las universidades como entidades formadoras y sus clientes.

6. Información extraída de: http://epp.eurostat.ec.europa.eu/tgm/refreshTableAction.do?tab= table\&plugin $=1 \&$ pcode $=$ teilm020\&language $=$ en .

7. http://sociedad.elpais.com/sociedad/2012/06/08/actualidad/1339157851_474635.html. 
Los nuevos sistemas de acceso a la Universidad establecidos en la legislación han sido implementados de diferentes formas en las universidades españolas. Nuestro equipo de investigación diseñó unos procedimientos concretos para el acceso que posteriormente fueron adoptados por todas las universidades públicas andaluzas. En este trabajo se han presentado las características básicas de estos procedimientos, así como los resultados de la primera convocatoria desarrollada en el curso 2009/10. La principal novedad introducida en estos nuevos sistemas de acceso es el reconocimiento de las competencias adquiridas por vías informales y no formales. Ello supone la diversificación de las vías de acceso al sistema educativo formal, y en parte también, la ampliación de las opciones formativas a aquellas personas que abandonaron tempranamente el sistema educativo. Con ello, se promueve la formación a lo largo de la vida, se mejora la cualificación de la población activa a través del acceso a la Universidad y se facilita el desarrollo profesional de los candidatos pudiendo contribuir asimismo a la mejora del mercado laboral.

Los sistemas de acceso que hemos diseñado han tenido una magnífica puesta en marcha funcionando de manera ágil y viable, remitiéndonos al número de candidatos que se han presentado y aprobado, y han podido acceder a alguna titulación. Esta valoración se sustenta con los datos aportados.

Las dificultades detectadas en el proceso de diseño y aplicación de estos sistemas han estado relacionadas, en primer lugar, con los plazos de elaboración de normativa, y la puesta en marcha del proceso en sí, que fueron muy ajustados en su primera convocatoria. En segundo lugar, la tabla que se construyó para relacionar titulaciones universitarias con la experiencia profesional que presentaban los candidatos era deficitaria en algunos aspectos. Concretamente, algunas profesiones no se relacionaron con algunas titulaciones que debían haber sido tenidas en cuenta, y al revés; de ahí que se haya realizado una revisión a fondo de estas correspondencias para la segunda convocatoria. Otra de las modificaciones que se ha llevado a cabo es la revisión de las "afinidades" relativas a la experiencia laboral, de manera que la valoración de la experiencia se realice en función de las ramas de conocimiento y las familias profesionales y no de titulaciones concretas. Con ello se ha logrado que las "acreditaciones" de acceso que obtienen los candidatos no caduquen y que además, no se vean afectadas por la modificación continua del mapa de titulaciones debido a la incorporación de nuevos títulos de Grado.

La demanda de acceso a través de estos nuevos procedimientos por parte de los adultos que desean mejorar su formación ha sido muy alta. Aunque inicialmente creíamos que estas pruebas podían restar candidatos a la de mayores de 25 años, los resultados de la primera convocatoria indican justamente lo contrario, reforzando la tendencia que comentábamos anteriormente (Rué, 2003-04; Pérez Díaz, 2001; Trinidad, 2005).

Los resultados de esta primera convocatoria parecen indicar un mejor rendimiento en el acceso para mayores de 40 años que en el acceso para mayores de 45 años. Ello puede deberse a que para superar la prueba de mayores de 40 años era necesario poseer una determinada experiencia laboral, y este requisito era público y fácilmente verificable antes de inscribirse en este procedimiento. Por tanto, era esperable que la gran mayoría de los presentados a estas pruebas las superaran. En cambio, para superar la prueba de mayores de 45 años era necesario obtener una nota mínima en 
dos exámenes, lo cual probablemente implicaba un mayor esfuerzo personal en la preparación de las pruebas y una mayor incertidumbre acerca de la superación de las mismas. En cualquier caso, la tasa de éxito en las pruebas para mayores de 45 de la Universidad de Huelva ha sido alta, lo cual puede deberse a que tanto nuestra universidad como algunos centros de adultos organizaron cursos de preparación para la superación de las mismas. Para el conjunto de las universidades andaluzas, sin embargo, los resultados de estas pruebas no fueron homogéneos.

Respecto a los candidatos presentados en Huelva en cada uno de los sistemas, existen algunas características que tienen una distribución muy similar en ambos grupos: género, edad y familia. Es interesante destacar que un $40 \%$ de los presentados a las pruebas de 45 años no hayan utilizado la vía de 40, a pesar de cumplir los requisitos para ambos procesos. Las posibles explicaciones pueden ser que su experiencia no se correspondía con la titulación que deseaban estudiar, no tener la suficiente experiencia en "tiempo", o simplemente preferir las pruebas escritas y no tener que preparar la documentación requerida para el otro sistema por ser muy laboriosa en algunos casos.

Existen diferencias interesantes entre ambos grupos respecto a las motivaciones. Parece que los participantes del sistema de 45 años podrían tener una motivación más intrínseca respecto al estudio: no les interesa tanto el prestigio y dicen querer estudiar para mejorar personalmente, estar activos y aprender... Esta motivación por aprender es identificada en trabajos como el de Salanova, Martínez, Bresó, Llorens y Grau (2005) como un agente facilitador del éxito académico en estudios. En dicha investigación se constata la existencia de una relación positiva entre los facilitadores en el estudio y el engagement ${ }^{8}$, compromiso, autoeficacia, satisfacción y felicidad relacionada con los estudios. El concepto de engagement es descrito relacionando tres aspectos: vigor, dedicación y absorción, que son medidos por tres escalas multi-ítem internamente consistentes y estables a lo largo del tiempo. Las expectativas de éxito son altas en ambos colectivos de mayores, siendo más optimistas los de 45 años. Estas diferencias pueden deberse a que el sistema de acceso de mayores de 45 incluye una prueba de evaluación, por lo ya tienen un referente de evaluación del ámbito universitario. Estas percepciones sobre su autoeficacia y la orientación intrínseca a la tarea (deseo por aprender) son identificados en los estudios de Martínez y Galán (2000) y en el de Cardozo (2008), como factores condicionantes del éxito académico. En otros trabajos sin embargo, como el de Valle, González, Nuñez, Rodríguez y Piñeiro (1999), en el que proponen un modelo causal sobre los determinantes cognitivos-motivacionales del rendimiento académico, llegan a afirmar en función de los resultados hallados, que alumnos con deseo de aprender y autoconcepto positivo (altas expectativas de logro) no generan necesariamente buenos resultados académicos. En cualquier caso, estos resultados también pueden deberse al grado de deseabilidad social con el que contestan a la encuesta, de ahí que nos planteemos hacer un seguimiento en este sentido en próximos trabajos de investigación.

8. Nos planteamos realizar un estudio para confirmar esta tendencia en futuros trabajos. 
Es destacable que tanto en el grupo de mayores de 40 como de 45, tengan pocos conocimientos sobre las titulaciones, sus planes de estudio o niveles de exigencia. Esta falta de información podemos asociarla a su desvinculación de los sistemas formativos oficiales, así como al momento de reforma universitaria en el que nos hallamos. Sin embargo, sí conocen las salidas profesionales probablemente derivado de su experiencia profesional. En cualquier caso, sería interesante ver si esas salidas son coincidentes con las establecidas en los grados y abrimos una nueva necesidad respecto a los servicios de orientación y tutoría de nuestras universidades, demandados por los colectivos de ambos sistemas de acceso (aunque mayoritariamente los del sistema de 40 años). Gairín, Feixas, Franch, Guillamón y Quinquer, (2003-2004) en este sentido, nos recuerda su importancia en los procesos de adaptación y rendimiento de nuestro alumnado, y en el caso de nuestros "mayores" serán casi con toda probabilidad, uno de los colectivos más necesitados. Por ello creemos interesante también hacer un seguimiento de estos colectivos tanto para centrarnos en el rendimiento y éxito académico, como para conocer su evolución respecto a la motivación e interés por los estudios.

Las valoraciones que los candidatos de ambos grupos han hecho sobre los sistemas de difusión de las convocatorias y de los sistemas propiamente dichos son altamente positivas. En nuestra opinión, la inexistencia de estas vías de acceso en la historia de la universidad española hasta esta convocatoria, podría estar en la base de las evaluaciones positivas con la que han recibido el proceso de acceso. Y esto a pesar de que desde la propia organización detectábamos como deficiencia la insuficiente difusión debido a la falta de tiempo entre el diseño y puesta en marcha de estos procesos y su celebración.

Además, los candidatos han considerado justos, transparentes y coherentes los sistemas de baremación, los exámenes y las entrevistas. Hay que decir que los sistemas diseñados en España han sido muy heterogéneos en el caso del acceso para mayores de 40 años. En cambio, el modelo andaluz establece de forma muy concreta los diferentes aspectos que deben ser valorados. Con objeto de evaluar la validez del modelo, nos planteamos un análisis comparativo con otros modelos implantados en España en las próximas convocatorias.

Uno de los aspectos más positivos de la introducción de estos nuevos sistemas de acceso es la diversificación del alumnado con la entrada en la Universidad de un mayor número de alumnos mayores. Esto va a suponer probablemente un enriquecimiento de nuestras aulas, sirviendo en ocasiones como una vía de conexión con el mundo laboral, e incluso como apoyo a los docentes aportando sus experiencias, y su bagaje profesional y personal en las aulas.

Para concluir quisiéramos señalar que la implantación de estas nuevas vías de acceso representan uno de los muchos retos a los que se enfrenta la Universidad en nuestros días, en este caso, se trata de uno de los retos que más beneficios puede aportar a nuestra sociedad, ya que, por una parte los ciudadanos encuentran una nueva posibilidad para completar su formación en un ámbito de estudio relacionado con su experiencia práctica de muchos años, y por otra, la Universidad consigue atraer a sus aulas a personas cuya formación superior había quedado interrumpida por la incorporación al mercado laboral, en cuyo seno y a través de formación no formal e informal, han adquirido competencias que ahora le suponen la posibilidad de retorno a estudios de nivel universitario. 


\section{REFERENCIAS BIBLIOGRÁFICAS}

ANECA (2008). Libros Blancos. Disponible en: http://www.aneca.es/Documentos-ypublicaciones/Libros-Blancos. [Consultado: 16-11, 2009].

AUSUBEL, D. (1981). Psicología educativa. Un punto de vista cognoscitivo. México: Trillas.

CARDOZO, A. (2008). Motivación, aprendizaje y rendimiento académico en estudiantes del primer año universitario. Laurus, 14 (28), 209-237.

COMISIÓN EUROPEA (2003). Education and Training 2010. The success of the Lisbon strategy hinges on urgent reforms. Brussels: Commission 11/11/2003. Disponible en: http://ec.europa.eu/education/policies/2010/doc/jir_council_final.pdf. [Consultado: 20-05, 2009].

ESCUDERO ESCORZA, T. et al. (1997). Las pruebas de acceso a la universidad. Revista de educación. Monográfico, 314.

GAIRÍN, J.; FEIXAS, M.; FRANCH, J.; GUILLAMÓN, C. y QUINQUER, D. (20032004). Elementos para la elaboración de planes de tutoría en la Universidad. Contextos Educativos, 6-7, 21-42. Disponible en: http://dialnet.unirioja.es/descarga/ articulo/1049447.pdf.

GONZÁlEZ, J. y WAGENAAR, R. (Eds.). 2003. Tuning Educational Structures in Europe. Bilbao: Universidad de Deusto.

INSTITUTO NACIONAL DE CUALIFICACIONES PROFESIONAL (INCUAL). Catálogo de cualificaciones. Disponible en: http://www.educacion.es/educa/incual/ ice_incual.html. [Consultado: 22-11, 2009].

INSTITUTO NACIONAL DE ESTADÍSTICA (2011). Encuestas de población activa 2011. Tercer Trimestre de 2011. Disponible en: http://www.ine.es/daco/daco42/ daco4211/epa0311.pdf. [Consultado: 11-10, 2012].

INSTITUTO NACIONAL DE ESTADÍSTICA (2012). Encuesta de población activa. Tasas de paro por nivel de formación alcanzado, sexo y grupo de edad. Disponible en: http://www.ine.es/jaxiBD/tabla.do. [Consultado: 11-10, 2012].

JUNTA DE ANDALUCÍA (2010). Resolución de 16 de febrero de 2010, de la Comisión del Distrito Único Universitario de Andalucía, por la que se establece el procedimiento para la obtención de los requisitos que permitan el acceso a los estudios de Grado de las Enseñanzas Universitarias de personas mayores de 40 y 45 años que no posean otros requisitos para el acceso a la Universidad. BOJA 57 de 23.03.2010.

MARTÍNEZ, J. R. y GALÁN, F. (2000). Estrategias de aprendizaje, motivación y rendimiento académico en alumnos universitario. Revista Española de Orientación y Psicopedagogía Vol. 11, 19, 35-50.

MINISTERIO DE EDUCACIÓN (2007). Ley Orgánica 4/2007, de 12 de abril, por la que se modifica la Ley Orgánica 6/2001, de 21 de diciembre, de Universidades. BOE 89 de 13.4.2007.

- (2008). Real Decreto 1892/2008, de 14 de noviembre, por el que se regulan las condiciones para el acceso a las enseñanzas universitarias oficiales de grado y los procedimientos de admisión a las universidades públicas españolas. BOE 283 de 24.11.2008. 
MINISTERIO DE EDUCACIÓN (2010). Panorama de la educación. Indicadores de la OCDE 2010. Informe Español. Madrid: Secretaría de Estado de Educación y Formación Profesional. Dirección General de Evaluación y Cooperación Territorial. Instituto de Evaluación.

- (2009). Catálogo Nacional de Cualificaciones Profesionales. Disponible en: http://www.educacion.es/educa/incual/ice_catalogoWeb.html. [Consultado: 2011, 2009].

MINISTERIO DE EDUCACIÓN Y CONSEJO ESCOLAR DEL ESTADO (2010). Informe sobre el estado y situación del sistema educativo. Curso 2008-2009. Madrid: Secretaría General Técnica.

MUÑOZ VITORIA, F. (1993). El sistema de acceso a la Universidad en España. 1940-1990. Madrid: Ministerio de Educación y Ciencia.

MUÑOZ VITORIA, F. (1995). El acceso a la Universidad en España: Perspectiva histórica. Revista de Educación, 308, 31-63.

PÉREZ DÍAZ, J. (2001). La actividad en las edades maduras y la salida de actividad. En Centre d'Estudis Demogràfics (CED) ¿Crecerán las tasas de actividad en Europa del Sur? Pronósticos a partir de una aproximación sociodemográfica. 2000-2001. Informe I+D encargado por la Comisión Europea (pp. 29-62). Madrid: Dirección General V Unidad V/E/1 (Empleo, relaciones laborales y asuntos sociales).

PINTRICH, P. R. y SCHRAUBEN, B. (1992). Students' motivational beliefs and their cognitive engagement in classroom academic tasks. En D. H. Schunk y J. Meece (Eds.) Students perceptions in the classroom. Hillsdale, N.J.: Lawrence Erlbaum (pp. 149-183).

ROCES, C.; TOURÓN, J. y GONZÁLEZ, M. C. (1995). Motivación, estrategias de aprendizaje y rendimiento de los alumnos universitarios. Bordón, 47, 107-120.

RUÉ, J. (2003-2004). Cambian los tiempos, pero ¿cambian las universidades? La Educación Superior ante los retos de la nueva sociedad. Contextos Educativos, 6-7, 167-186. Disponible en: http://dialnet.unirioja.es/servlet/fichero_articulo?codigo= 1049481.

TRINIDAD, A. (dir.) (2005). La Educación superior en Andalucía. Evolución y posición en España. Madrid: Editorial Tecnos.

SALANOVA, M.; MARTÍNEZ, I.; BRESÓ, E.; LLORENS, S. y GRAU, R. (2005). Bienestar psicológico en estudiantes universitarios: facilitadores y obstaculizadores del desempeño académico. Anales de Psicología, 21, 170-180.

VALLE, A.; GONZÁLEZ, R.; NÚÑ̃EZ, J. C.; RODRÍGUEZ, S. y PIÑEIRO, I. (1999). Un modelo causal sobre los determinantes cognitivo-motivacionales del rendimiento académico. Revista de Psicología General y Aplicada, 52 (4), 499-519.

ZABALZA, M. A. (2003-2004). La innovación en la enseñanza universitaria. Contextos Educativos, 6-7, 113-136. Disponible en: http://dialnet.unirioja.es/descarga/ articulo/1049473.pdf. 


\section{ANEXO}

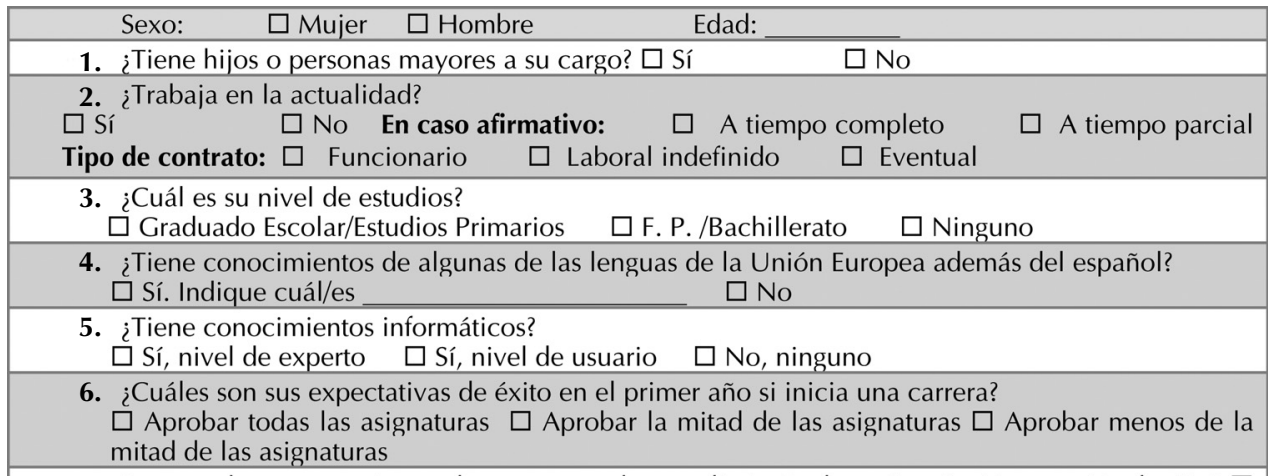

7. En caso de superar esta prueba, ¿conoce el procedimiento de preinscripción y matriculación? $\square$ Sí $\quad \square$ No

8. ¿Qué le ha motivado a presentarse a las pruebas de acceso a la Universidad y así tener la opción de iniciar estudios universitarios?

- Mejorar mis opciones profesionales (promoción; acceso a puestos distintos, acceder al mercado laboral,,.. )

- Profundizar conocimiento

- Crecimiento personal

- Estar activo/a mentalmente

- Establecer nuevas relaciones de amistad

- Siempre quiso estudiar

- Consejo de familiares y amigos

- Prestigio social de poseer una titulación universitaria

Si cree que estudiar una Titulación Universitaria le reporta otro/s beneficio/s, especifíquelos

9. La Titulación a la que le gustaría acceder:

- Está relacionada con su actual campo de trabajo o con empleos anteriores

- La ha elegido porque le gustaría trabajar en ese campo profesional

- Tiene muchas salidas profesionales

- Le permite promocionar en su actual empleo

- Es fácil y tiene una duración adecuada

- Le gusta y la cursaría como desarrollo personal

10. Valore el nivel de información que posee acerca de los estudios que le gustaría cursar

- Conoce el plan de estudios de la titulación

- Conoce el nivel de exigencia

- Conoce las salidas profesionales

11. Valore la información que ha recibido por los siguientes medios para inscribirse en el proceso de ACCESO para mayores de 40 años

- Medios de comunicación

- Web de la Universidad

- Internet en general

- Orientación de personal de la Universidad

- Especifique por favor, si ha recibido información por otros medios:

12. Valore aspectos de organización y gestión del procedimiento de Acceso para mayores de 40 años

- El proceso de inscripción está bien organizado

- La información inicial del procedimiento para el acceso (requisitos, documentación a aportar, fechas de entrevistas, ...) es adecuada

- Considera que habría que crear un servicio de orientación para este acceso

- La tabla de afinidades entre Experiencia Profesional y Titulaciones Universitarias que dan

la posibilidad de acceder a una carrera universitaria me parece adecuada.

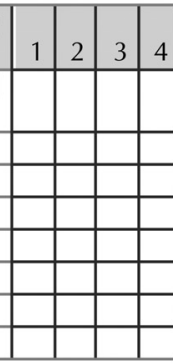

En caso que haya valorado el ítem anterior negativamente (valoración 1), indique las carreras asociadas a

Titulaciones que cree que deberían estar recogidas: 


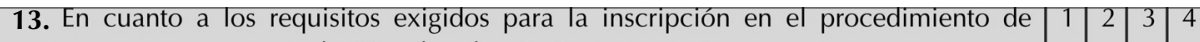
acceso para mayores de 40, valore los siguientes aspectos

- Las personas encargadas del procedimiento administrativo han atendido las dudas que hayan podido surgir

- El sistema de baremación empleado (tiempo de experiencia y competencia profesional) me parece muy adecuado

- Las entrevistas como prueba de evaluación de la madurez del candidato me parece muy adecuado

14. ¿Qué opinión le merece que se haya flexibilizado el acceso a la universidad incorporando estas dos nuevas vías para mayores de 40 y 45 años?

15. Si tiene alguna propuesta de mejora respecto al procedimiento de acceso, por favor, especifíquela.

Muchas gracias por su amabilidad al contestar nuestro cuestionario y por prestarnos su ayuda en nuestra investigación. 
